\title{
Analysis on Influencing Parameters of Heating Consumption Prediction
}

\author{
Ye $\operatorname{Tian}^{1}, \mathrm{Rui}^{\mathrm{Li}^{1 *}}$ \\ ${ }^{1}$ School of Environment and Energy Engineering, Beijing University of Civil Engineering and Architecture, Beijing 100044, China
}

\begin{abstract}
This paper studies the influencing parameters of the heating consumption prediction in heating substation, uses the BP neural network to predict the heating consumption, and establishes four BP neural network structures to change the outdoor average temperature, the lowest temperature and the highest outdoor temperature, the predicted results found that when the input variables include the average outdoor temperature, the lowest outdoor temperature, the highest outdoor temperature, the wind speed, and the heating consumption of the previous three days, the prediction results are better, the relative error is equal or less than $0.25 \%$.
\end{abstract}

\section{Introduction}

The accuracy of heating consumption of district heating system affects the heating effect and economy of the heating system operation. It shows that some of the existing prediction methods are not accurate enough, and sometimes heating consumption is higher than the demand, and sometimes can not meet the needs of users. When the heating consumption is higher than the demand, a lot of energy will be wasted. Therefore, finding a mor accurate and usable model is of significance to the energy saving of district heating.

As a new type of prediction method, artificial neural network prediction has been used by many scholars in the research of heating consumption prediction. $\mathrm{Li}$ et al. analysed the influencing parameters and prediction methods of heating substation, and found that the influencing parameters of heating consumption are not only related to outdoor temperature, wind speed and other climatic conditions, but also related user types, user heat characteristics, building age. The heating consumption of heating substation is affected by multiple parameters and has non-linear characteristics [1]; Zhu et al. studied the prediction of BP neural network in heating energy saving and found that the prediction of BP neural network greatly reduces the operating energy consumption of the district heating system [2].

It can be seen that the neural network prediction method is effective for heating consumption prediction, but it has not yet reached to applicable. This paper analyses the influencing parameters of the heating consumption of district heating system, and establishes a model. Through the comparison between models, a more accurate heating consumption prediction model can be obtained.

\section{Prediction methods and models}

Currently commonly used prediction methods include time series prediction method, gray prediction method, ANN prediction method and neural network prediction method, etc. Artificial neural network method does not need to establish a complex relationship between input and output, and is processed by using a set of weights. Non-linear problems can realize the mapping of input and output. The model established by this method is more reasonable than other methods in the treatment of nonlinear problems, effectively improves the accuracy, and is more suitable for short-term heating consumption prediction [3].

Artificial neural network is a mathematical model that uses a structure similar to the synaptic connections of the brain to perform information processing. It is an abstraction, simplification and simulation of the human brain, reflecting the basic characteristics of the human brain. The research of neural network is to study human's intelligent behaviour from the physiological structure of human brain, and simulate the function of human brain to process information. According to different structures, functions and learning algorithms, neural networks can be divided into perceptron neural networks, linear neural networks, BP neural networks, radial basis neural networks, and so on. Among them, BP neural network is the most widely used network, which has a multi-layer hidden structure and contains one or more hidden layers. $\mathrm{BP}$ refers to the method of signal forward propagation and error back propagation during gradient calculation in nonlinear multilayer network training.

\subsection{Prediction models}

Build a two-layer feedforward BP neural network with sigmoid neurons and linear output Neurons, as shown in 
Figure 1.

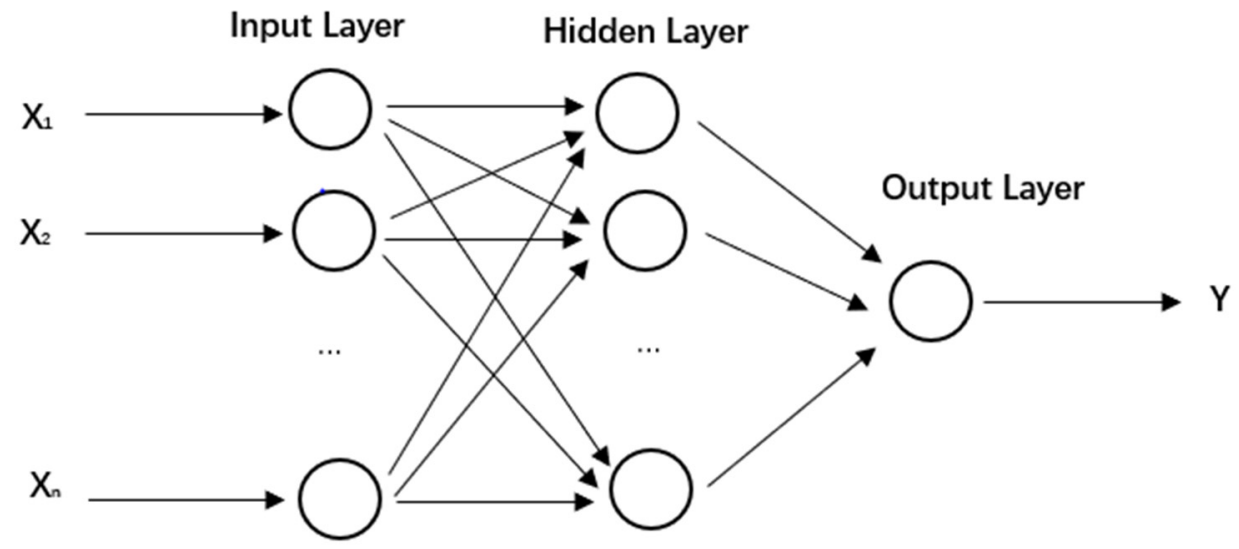

Figure 1. A two-layer feedforward BP neural network.

There is no exact method to determine the number of neurons in the hidden layer, so it is determined according to the empirical Eq.(1),

$$
\begin{aligned}
& m \\
& =\sqrt{l+n}+a
\end{aligned}
$$

Where $l$ is the number of neurons in the input layers, $n$ is the number of neurons in the output layers, $m$ is the number of neurons in the hidden layers, $a$ is a constant between 1-10[4].

The input sample is a total of 83 sets of data of winter in 2018 , of which $70 \%$ are used for training, $15 \%$ are used for verification, and $15 \%$ are used for testing. Using Levenberg-Marquardt as the algorithm of the neural network, training automatically stops when generalization stops improving, as indicated by an increase in the mean square error of the validation samples.

The common input parameters of the four models are wind speed and heating consumption in the previous three days. In addition, they have different input parameters. The input parameters of model $\mathrm{A}$ are outdoor average temperature and minimum temperature; the input parameters of model B are outdoor average temperature; the input parameters of model $\mathrm{C}$ are outdoor average temperature and maximum temperature; the input parameters of model D are outdoor average temperature, minimum temperature and maximum temperature.

\subsection{Analysis of Influencing parameter}

\subsubsection{Climatic parameters}

Climatic parameters are necessary parameters that affect heating consumption, including temperature, humidity, pressure, wind speed, wind direction, etc. Analyse the daily data of a heating substation in a residential area in Beijing. Figure 1 shows the variation curve of outdoor temperature and heating consumption, and Figure 2 shows the variation curve of wind speed and heating consumption. It can be seen that there is a certain correlation between outdoor temperature and wind speed with heating consumption. In most days, outdoor temperature and wind speed are negatively correlated with heating consumption. Therefore, take outdoor temperature and wind speed as a parameters that predicts heating consumption.

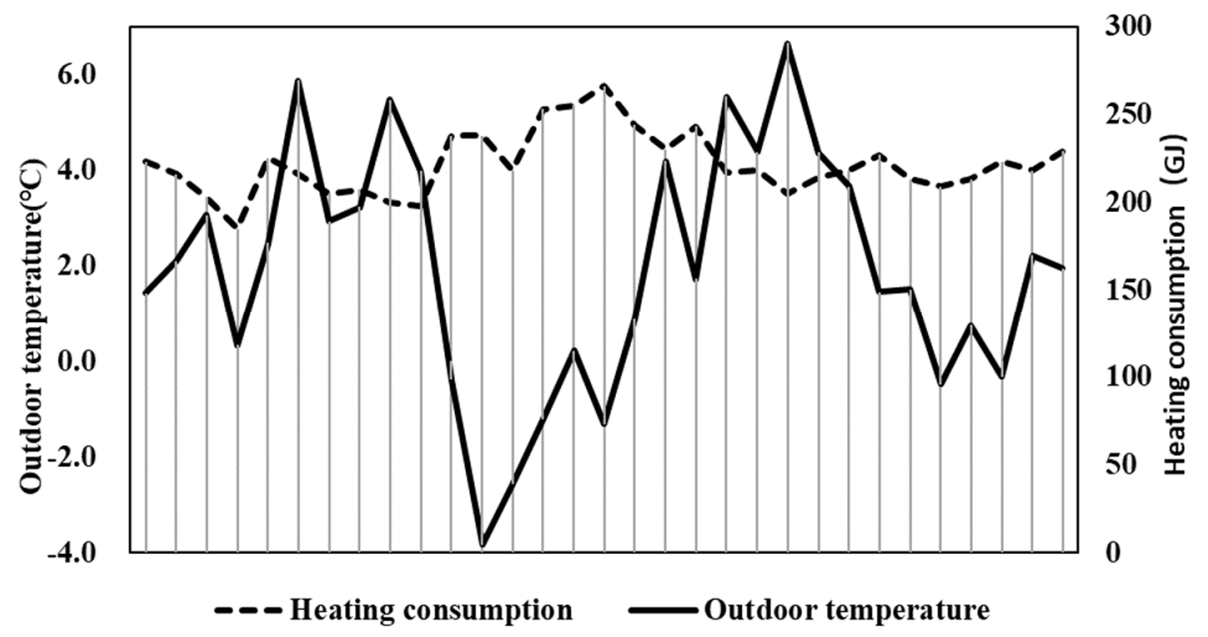

Figure 2. Variation curve between outdoor temperature and heat consumption. 


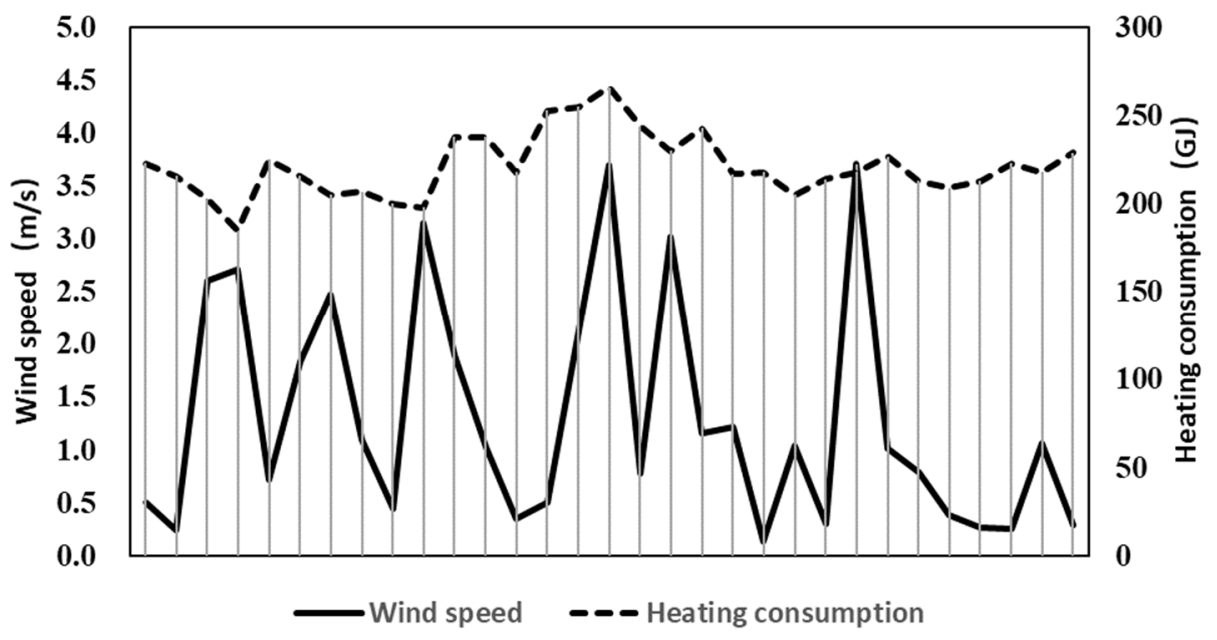

Figure 3. Variation curve between wind speed and heat consumption.

\subsubsection{Architectural parameters}

Due to the thermal inertia of the envelop configuration of buildings, there is an attenuation and delay relationship between the fluctuation range of the heat transfer and temperature through the envelope structure and the external disturbance fluctuation range. The degree of attenuation and hysteresis depends on the heat storage capacity of the envelope structure. The greater the heat capacity of the envelope structure, the greater the heat storage capacity, the longer the lag time, and the greater the attenuation of the wave amplitude. Therefore, it is necessary to add the heating consumption for the first three days of the residential area to the model.

\section{Results and Discussion}

The neural network system is used to simulate the above four models, as shown in Figure 3, which is the difference between the actual value and the predicted value of the 7 samples. It can be seen that the error of Model D is smaller than the other three models, most of them are within $0.25 \%$. Calculate the average error of 83 samples. Model $\mathrm{A}$ is $0.54 \%$, model $\mathrm{B}$ is $1.57 \%$, model $\mathrm{C}$ is $3.01 \%$, model $\mathrm{D}$ is $0.25 \%$, and model $\mathrm{D}$ has the smallest error, which also means that its prediction accuracy is higher than the other three Models.

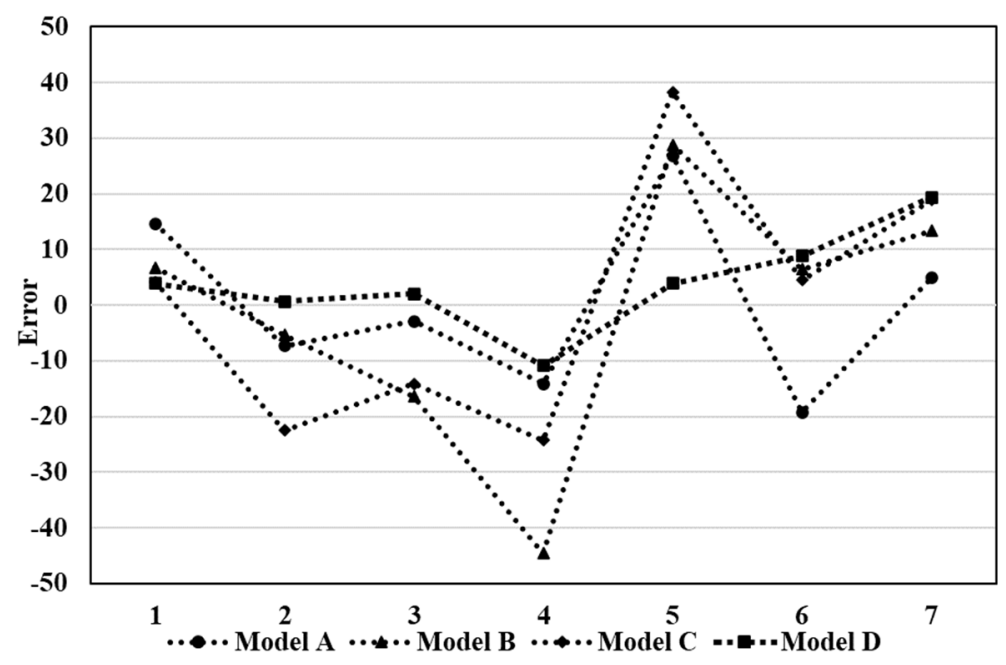

Figure 4. The error of model A, B, C and D.

\section{Conclusions}

This paper analyses the parameters affecting the heating consumption of a heating substation in a residential area, and establishes a prediction model using BP neural network, and the conclusions are as follows:

- $\quad$ Outdoor temperature and wind speed are roughly negatively related to heating consumption, indicating that outdoor temperature and wind speed are necessary parameters for predicting heating consumption. 
- In addition to meteorological parameters, considering the thermal inertia of the building, the heating consumption of the previous three days is also added to the prediction model.

- $\quad$ Use BP neural network method to predict heating consumption, add wind speed and heat load of the previous three days to the model, and then establish four models: the input parameters of model A are the average outdoor temperature and the minimum temperature; the input parameters of model B Is the average outdoor temperature; the input parameters of model $\mathrm{C}$ are the average outdoor temperature and the maximum temperature; the input parameters of model D are the average outdoor temperature, the minimum temperature and the maximum temperature, and the error of model D is the smallest. Therefore, the average outdoor temperature, minimum temperature and maximum temperature are all important that affect the heating consumption. Inputting the prediction model can make the prediction more accurate.

\section{Appendices}

This paper was supported by 'Beijing Advanced Innovation Center For Future Urban Design, Beijing University Of Civil Engineering And Architecture' (UDC2019011121).

\section{References}

1. Li, R., Hao, X.J., Fu, B. Wang, L.X., Sun, Z.Y., Dong, Y.(2018) Analysis and Prediction Method of Influencing Parameters of Heating consumption of Heating subsubstation. J. District heating, 25-29.

2. Zhu, D.X., Lu, S.H., Zhang,F., Guo, J.W., Ge, X.F.(2020) Energy-saving application of BP algorithm predictive control in heating system. J. Building energy \& Enviroment, 39:50-52.

3. Zhang, M.L., Liu, S.G. (2018) Research on Heating consumption Forecasting Method of District Heating System. J. Shanxi Architecture, 120-121.

4. Li, H.D., Guan, D.X., Yuan, F.H. (2015) BP artificial neural network simulation of poplar canopy transpiration. J. Acta Ecologica Sinica, 4137-4145. 